\title{
Rational Instability in the Natural Coalition Forming
}

\author{
Galina Vinogradova* \\ CREA - Center of Research in Applied Epistemology, Ecole Polytechnique, 32 boulevard Victor \\ Paris, 75015, France \\ Serge Galam ${ }^{\dagger}$ \\ CREA - Center of Research in Applied Epistemology, Ecole Polytechnique, 32 boulevard Victor \\ Paris, 75015, France
}

\begin{abstract}
We are investigating a paradigm of instability in coalition forming among countries, which indeed is intrinsic to any collection of individual groups or other social aggregations.

Coalitions among countries are formed by the respective attraction or repulsion caused by the historical bond propensities between the countries, which produced an intricate circuit of bilateral bonds. Contradictory associations into coalitions occur due to the independent evolution of the bonds. Those coalitions tend to be unstable and break down frequently.

The model extends some features of the physical theory of Spin Glasses. Within the frame of this model, the instability is viewed as a consequence of decentralized maximization processes searching for the best coalition allocations. In contrast to the existing literature, a rational instability is found to result from forecast rationality of countries.

Using a general theoretical framework allowing to analyze the countries' decision making in coalition forming, we feature a system where stability can eventually be achieved as a result of the maximization processes. We provide a formal implementation of the maximization principles and illustrate it in the multi-thread simulation of the coalition forming. The results shed a new light on the prospect of searches for the best coalition allocations in the networks of social, political or economical entities.
\end{abstract}

Keywords: Coalitions Forming, Social Modeling, Social Simulation, Political Instability, Economical Instability, Statistical Physics.

\footnotetext{
*galina.vino@gmail.com

†serge.galam@polytechnique.edu
} 


\section{Introduction}

The subject of our study is the phenomena of instability in coalition forming intrinsic to any collective of individual groups. To illustrate the presentation keeping it close to topical interest, we address the coalition forming in an aggregate of countries. However, the discussion and the results can be applied to any type of collective in political, social or economical sciences.

For centuries, countries have been undergoing parallel processes of association into unities seeking mutual support and cooperation, and fragmentation of the unities due to the bilateral conflicts. Association and fragmentation are driven by the character of relationships and interactions built by the countries through the history. These interactions have formed very strong bilateral mutual propensities between the countries, the propensities to cooperate or to conflict.

These propensities are the key factors that determine the association into coalitions. Like in the well known saying "the enemy of an enemy is a friend", we assume only two competing coalitions. Guided by the postulate of lowest energy, we expect that positive propensities encourage countries to ally to the same coalition, while negative ones encourage them to affiliate with the opposing coalition. Thus, the best coalition allocation - the one where the countries have most comfortable and beneficial position, is achieved in an amalgam of conflict and cooperation. Cooperation between countries maximizes their benefit (minimizes their local energy) in case of positive propensity. Negative propensity, on the contrary, minimizes the benefit of cooperation, while it maximizes the benefit of conflict.

In the model of countries collective we assume that no external forces incite the interactions between the countries. Consequently, the coalitions are formed only through the attraction or repulsion caused by the historical bond propensities between the countries.

Spontaneous and independent evolution of the countries mutual propensities produce a priori a very intricate circuit of bilateral bonds. They are the cause of contradictory association within the coalitions which may include conflicting countries brought together attracted by common allies. Such associations tend to be unstable, they break down frequently, forming new associations.

The nominal model is referred as natural model of coalition forming. The model resembles the

Statistical Physics model of Spin Glasses (Binder \& Young 1986), which is an idealized model of bulk magnetism represented by a collection of interacting spins - atoms acting as a tiny dipole magnet with a mixture of ferromagnetic and anti-ferromagnetic couplings. The countries are compared to the magnetic dipoles which interact with each other and align themselves in order to attain the most comfortable position. The collection of spins forms a disordered material in which the competing interactions cause high magnetic frustration - changes of spins with no energy cost.

In order to reproduce more realistically a system of individual actors that possess rationality, we suppose the countries observe and make decision before processing to any change. This provides a basis to a rational instability, which is not only the no-cost frustrations appropriate to the spins, but also the cost frustrations driven by the future benefit of planned changes. Rationality of the 
instability makes the main difference between the Spin Glass and the present model.

Spin Glass formulations in Statistical Physics are set out in (Binder \& Young 1986) and (Toulouse 1977). The modeling of complex social situations using Statistical Physics has started from (Galam \& Gefen \& Shapir 1982). However, the subject of the instability and the stabilization in coalition forming using the model is rather recent. The authors of Galam \& Moscovici 1991) study a model of collective decision making combining Social Psychology hypotheses with recent concept of Statistical Physics. Then, the coalition as a form of aggregation among a set of actors (countries, groups, individuals) has been studied using concepts from the theory of Spin Glasses Axelrod \& Bennett 1998; Florian \& Galam 2000; Galam 1996, 1998, 2002; Gerardo \& Samaniego-Steta \& del Castillo-Mussot \& Vazquez 2007; Tim Hatamian 2005; Matthews 2000).

The seminal paper (Axelrod \& Bennett 1998) applies the Spin Glass model to the aggregation and the alignment of actors. In (Galam 1996), this application has been shown to be insufficient to describe the instabilities of coalitions. The author extends the idea by combining both random-site and random-bond Spin Glass models. He, then proposes a political model allowing to reproduce the incompatible interactions in the formation of coalitions. Various social application of the model were suggested. For example, (Galam 2002; Gerardo \& Samaniego-Steta \& del Castillo-Mussot \& Vazquez 2007) use it to explain the formation of coalitions and suggest its social and political application. The dynamical analogue of the model and it's viability is discussed in (Vinogradova 2012).

There is also literature that studies social or inter-agents stabilization using a different class of models. (Antal \& Krapivsky \& Redner 2006) consider transition of social network to a balanced state by dynamical changes of sign of the mutual links, and (Acemoglu \& Egorov \& Sonin 2006) feature the formation of coalition by dynamical collective decision making.

The literature studying the coalition forming based on the Spin Glass model analyze the phenomena within a Markovian frame of spontaneous instability. In contrast, the current work assumes long horizon rationality of the actors to develop the rational instability framework of coalition forming. This allows to explore and study the complex behaviors among individual actors possessing rationality when they form coalitions.

Let us note that, while in Spin Glass model the Game Theory equilibrium represents a stable configuration of the spins, the equilibrium guarantees no stability in a model where the agents do not stop searching upon achieving their local maximums. Therefore, the game-theoretic tools are not appropriate for the stabilization objective of our model.

In this work, we extend the model proposed by (Galam 2002) and provide a detailed analysis of the instability rooted in the coalition forming as a decentralized maximization processes driven by each country's objective to attain its best coalition allocations. We study the terms of existence of the optimal and stable coalitions, and the ways to reach them in the ranges of extensive or limited rationality. This allows to shed a new light on the interesting and little explored phenomena such 
as information manipulation and non-optimal stability, which are peculiar to any network of selfish actors.

Among the results, the model provides an explanation of why in most cases in practice where the rationality prevails, the instability constantly follows the natural coalition forming.

The model's theoretical framework is supplemented by historical examples that justify the practical use of the model. We also provide the description and the illustration of a multi-threaded simulation of coalition forming in the natural model that is created for this particular aim. Finally, we provide concluding remarks and discuss the directions of the further study.

\section{Natural Model of Coalition Forming}

How does the historical and geographical background impact the current interactions between the countries? Based on their historical experience the countries decide weather to agree or disagree on the present policies. Respectively, the agreement is unfavorable to the countries which went thought of rejection. The same way, the disagreement is unfavorable to the countries that passed through a conflict in their past. The primary mutual propensities between countries are the issues of their historical experience so that their character of cooperation or conflict can hardly be changed. Those propensities affect all the subsequent interactions and exchanges between the countries.

Here, we present the natural model of coalition forming that describes a system of countries maintaining short range interactions which are guided by the primary mutual propensities.

Consider a group of $N$ independent countries which had experienced geographic, cultural or economic interactions during their history. The countries are respectively denoted by characters or indicators ranging from 1 to $N$. Each country thus makes its choice among two options +1 and -1 , corresponding to two possible coalitions. The same choice allies the countries to the same coalition, while different choices separate them into the opposite ones. According to the postulate of minimum conflict, being part of the same coalition benefits the countries with the propensities to cooperate, while countries inclined to conflict bear loses from the cooperation. A country is represented by a discrete variable that can assume one of the two state values $S=+1$

and $S=-1$. The combination $S=\left\{S_{1}, S_{2}, S_{3}, \ldots, S_{N}\right\}$ makes up the configuration of the choices of the countries. The configuration of choices, as well as it's inverse $S=\left\{-S_{1},-S_{2},-S_{3}, \ldots,-S_{N}\right\}$ by symmetry, represents a particular configuration of coalitions in the system.

Consider any two countries $i$ and $j$, and denote by $J_{i j}$ the value that measures the degree and the direction of the historical exchanges between the countries. $J_{i j}$ represents the bond of original propensity between the countries, which is symmetric, $J_{i j}=-J_{j i}$, and may vary for each pair of countries. $J_{i j}=0$ when no mutual bond exists between the countries, which represents an absence of a direct exchange. We shall describe by $J_{i j} S_{i} S_{j}$ the measure of the interaction between the countries as a function of their choices. In case the countries agree, i.e., when $S_{i}=S_{j}$, a positive value of $J_{i j}$ results in the positive effect for both the countries. When $J_{i j}$ is negative, the 
cooperation between the countries results in a negative effect on both the countries. That would agree with a widespread notion saying that conflicting countries gain from disoperation while incur losses from cooperation.

Thus, the propensities either favor the cooperation $\left(J_{i j}>0\right)$, the conflict $\left(J_{i j}<0\right)$, or signify the ignorance $\left(J_{i j}=0\right)$ where neither the agreement nor the disagreement influences the outcome of the countries.

For the sake of visualization, we represent the system of countries as a connected weighted graph with the countries in the nodes and the bilateral propensities as the weights of the respective edges, (see Figure (1D). We take red (dark) color for +1 choice and blue (light) color for -1 choice.

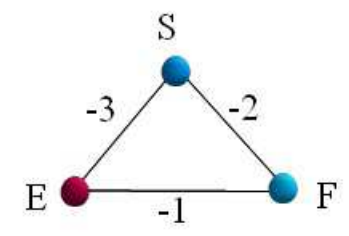

Figure 1: Triangle of three countries connected by negative bond propensities - ESF conflicting triangle.

Sum of the measures of all the interactions of a country $i$ in the system is the net gain of the country

$$
H_{i}(S)=S_{i} \sum_{j \neq i} J_{i j} S_{j}
$$

. The total gain in the system in configuration $S$ is measured by the total of the contributions in the configuration

$$
\mathcal{H}(\mathcal{S})=\frac{1}{2} \sum_{i} H_{i}(S)
$$

The natural model is formally identical to the Ising Model of Spin Glass in Statistical Physics. The model consists of $N$ discrete variables of magnetism $\left\{S_{i}\right\}_{1}^{N}$, called spins, that can be in one of two states up or down. See figure (21). The spins, arranged in a lattice or a graph interact at most with its nearest neighbors. Spins with the same states are associated to each other, and disassociated from those with the opposite states.

In this analogy, the total gain $\mathcal{H}(\mathcal{S})$ in the system is identical to the Hamiltonian of an Ising random bond magnetic system. The model of the Spin Glass has been solved numerically with much effort ((Binder \& Young 1986) $)$.

\subsection{Illustrating of The Natural Model Trough an Historical Example}

As an example of an unstable system we suggest to consider the typical conflicting triangle of Spain, England and France, which were alternatively enemies and allies during a long period of 


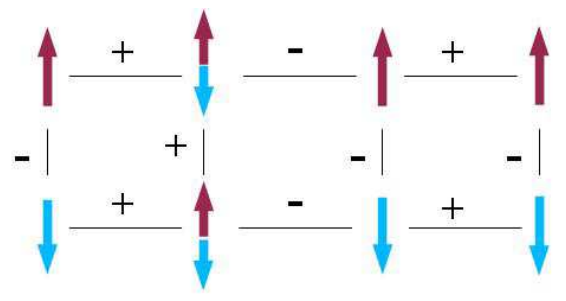

Figure 2: Ising model of 8-spins with negative pair interactions. Spins that shift their states with no loss of energy, called frustrated spins, are marked by both up and down the states. A Spin Glass in an unstable disorder.

time. For these countries, the period of 1521 - 1604 has been marked by the series of land and sea wars driven by the historical background of colonization, religion and by the naval technical progress. The commercial rivalry between England and Spain, and political and religious ambitions of France were the major forces that pushed Europe into wars. The conflicts were usually initiated between any two of the countries with the third one joining the one or the other side. Accordingly, the historical background of the countries during this period has defined the particular distribution of mutual negative propensities.

The natural model is shown schematically in the ESF conflicting triangle in Figure (11), where abbreviations $S, E$ and $F$ stand for the countries' names. The choices of the countries $S, E$ and $F$ are represented by the state variables $S_{E}, S_{S}$ and $S_{F}$ respectively. Different colors attached to the state nodes of the countries correspond to different coalitions the countries. The state nodes are linked by the following original propensities $J_{S E}=-3, J_{S F}=-2, J_{E F}=-1$. The value of propensities are illustrative only and this is their relative magnitudes are of importance: the conflict between Spain and France is less deep than the one between Spain and England, yet it is deeper than the one between England and France.

\section{$3 \quad$ Instability in Coalition Forming}

During the coalition forming, at any particular configuration, a country may observe that another configuration exists where it can reach a higher gain. When it happens, the country shall take appropriate changes in order to take advantage of this opportunity. The sequence of the changes constitutes the process of maximization of the countries' gains.

Maximization, which is a search for the most beneficial coalitions setting is found to produce an instability. When the countries eventually attain a common satisfactory configuration, the instability is temporary. When it is a permanent one, the maximization of the countries' gains is an endless process. 


\subsection{Geometric Terms of Instability}

Let us recall that in the natural model we extend the notion of instability of the physical systems where it is only caused from frustration of spins due to an immediate improvement or due to no cost of changing. In the broader sense, frustrations due to intended changes aimed to improve the current gains in further steps are also included. Such improvements are peculiar to the system of countries which are able to anticipate and adapt to the changes of others. Spins, unlike countries, are able to evaluate only the effect from the immediate changes. In order to be able to predict such an unstable character of a system of countries, we provide the necessary and sufficient conditions of the instability.

Let us take a close look at the heart of the instability. Consider separately from the rest of the system, two countries $i$ and $j$ connected by their propensity bond $p_{i j}$. When $p_{i j}$ is the only link between the countries, we can easily valuate the contribution from cooperation or conflict between the countries to their gain: $p_{i j} S_{i} S_{j}$. What complicates the evaluation of their alternative choices is the presence of the other countries connecting between $i$ and $j$ indirectly.

By a circle in a graph of countries we understand a selection of nodes linked into a closed path by the propensity bonds. Imagine a situation where the countries $i$ and $j$ is part of a circle and assume without loss of generality that their mutual propensity is negative while the propensities all along the rest of the circle are positive (see Figure (30). In this case, both $i$ and $j$ maximize their gain in conflict with the other and in cooperation with the rest countries on the circle. Such an arrangement creates an everlasting competition between $i$ and $j$ for this maximizing arrangement. The countries continuously shift their choices that produces the instability.

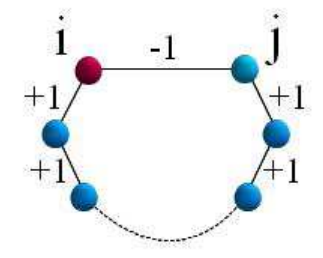

Figure 3: Circle connecting between the countries $i$ and $j$ by a negative and a positive links at the same time produces an everlasting competition between the countries.

We are now ready to identify the instability formally. Denote a circle of countries by $\mathcal{C}$ and the countries composing the circle by $1,2, \ldots, k$. Then the characteristics of the phenomena of instability in Spin Glasses (Toulouse 1977) can be interpreted in the terms of the natural model:

if there is a closed circle of countries on which the product of total propensities is negative,

$$
\Pi_{i, j \in \mathcal{C}} p_{i j}<0
$$

then the system is unstable. 
Terms (3) feature the instability in a system of countries as a direct consequence of geometry of the system - presence of a negative circle. In order to identify the instability, the terms require to examine all the possible circles in the system, while provide no countries' competition data. We call (3) geometric terms of instability.

The geometric instability terms, while originate from physics, provide only the necessary condition for the instability in the Spin Glass. The Spin glass with a negative circle can be stable when, for example, an interaction with a neighbor spin shifts the energy of the spin form zero to negative and thus keeps the spin from fluctuating. This case illustrated in Figure (4).

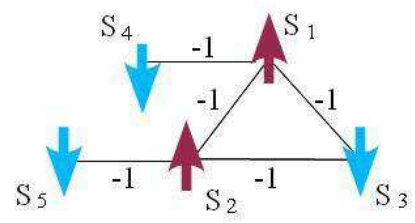

Figure 4: Configuration which is stable in Spin Glass and unstable in the system of countries.

In the extended sense of instability, (3) provides also a sufficient condition of instability, the one that induces the endless competition among the countries for beneficial coalitions.

\subsection{Optimal Configurations and Analytical Terms of Instability}

By making a choice, a country $i$ realizes one of two possible system's configurations $S^{+i}$ and $S^{-i}$ which differ by the country $i$ 's choice $S_{i}$. In order to select the preferable one, the country needs to classify the configurations by the order of preference based on the potential gain.

A preference order over a set of elements in general, is defined by a binary relation on the set which is a collection of ordered pairs of its elements. Each country $i$ 's function of gain $H_{i}$ sets the binary relation of preference $\leq_{i}$ over the set of all system's configurations as follows: configuration $S^{\prime}$ is less preferred than $S^{\prime \prime}, S^{\prime} \leq_{i} S^{\prime \prime}$ if $H_{i}\left(S^{\prime}\right) \leq H_{i}\left(S^{\prime \prime}\right)$. The total gain in the system $\mathcal{H}$, in turn, sets the relation $\leq_{\text {sys }}$ where $S^{\prime} \leq_{\text {sys }} S^{\prime \prime}$ if $\mathcal{H}\left(S^{\prime}\right) \leq \mathcal{H}\left(S^{\prime \prime}\right)$. Each of the relations $\left\{\leq_{i}\right\}_{i=1}^{N}$ and $\leq_{\text {sys }}$ defines a partial order over the set of configurations - the order that indicates a relation between some elements while others remain unrelated. For each particular partial order, those unrelated configurations yield the same gain to the country and therefore belong to the same equivalence classes. The equivalence classes thus are strictly ordered from minimal to maximal.

Each equivalence class, unifying under the same gain the configuration and its inverse, contains at least two configurations which a priori leaves only half of the possibilities of different gains, $2^{N-1}$.

The maximal configurations of a country are contained in its maximal equivalence class. Similarly, the system's maximal configurations are in the system's maximal equivalence class. Consequently, the common maximal configurations, which are those that satisfy all the countries, lie 
in the intersection of the maximal equivalence classes of all the countries. If we denote by $C_{i}^{\gamma_{i}}$ an equivalence class of country $i$ corresponding to gain $\gamma_{i}$, and by $C_{i}^{\Gamma_{i}}$ the maximal equivalence class, then the set of common maximal configurations is determined by

$$
\mathcal{S}^{\Gamma_{i}}=\cap_{i=1}^{N} C_{i}^{\Gamma_{i}}
$$

If $\mathcal{S}^{\Gamma_{i}} \neq \emptyset$, then the countries share the same maximal configuration in the system. The configuration is an optimal configuration, the one that satisfies all the countries and guarantees stability of the system.

Statement 1 Formula (4) provides new terms of the instability reading that if

$$
\cap_{i=1}^{N} C_{i}^{\Gamma_{i}}=\emptyset
$$

than the system is not stable in any configuration.

This is the analytical terms that, along with the indication of system's stability, provide particular optimal configurations, as well as the respective values of gains of the countries.

Here, the phenomena of instability is explained by the fact that, a priori, preference orders of different countries do not coincide, and consequently, maximal coalitions for one country is not maximal for the others.

Statement 2 Let us remark that when an optimal configuration exists, it is unique up to inverse.

Assume, by contradiction, that there are two different optimal configurations which however are not the inverse of each other, $\mathcal{O} 1$ and $\mathcal{O} 2$. Then, there is at least one country whose states differ in the two configurations, and another country whose states agree in the configurations. As far as we consider a connected system, there are necessarily two such countries which are connected by a propensity bond. Denote them by $i$ and $j$. Then, $S_{i}^{O 1}=-S_{i}^{O 2}$ and $S_{j}^{O 1}=S_{j}^{O 2}$.

Assume, without loss of generality, that $J_{i, j}=J>0$. Since $\mathcal{O} 1$ and $\mathcal{O} 2$ are both optimal then $\mathcal{H}_{i}(\mathcal{O} 1)=\mathcal{H}_{i}(\mathcal{O} 2)$. Since they differ by the states of $i$, there is country $k$ such that $J_{i, k}=-J$ and the following holds: $\mathcal{H}_{i}(\mathcal{O} 1)=\mathcal{H}_{i}(\mathcal{O} 2)-J_{i, j}+J_{i, k}$ and $\mathcal{H}_{i}(\mathcal{O} 2)=\mathcal{H}_{i}(\mathcal{O} 1)+J_{i, j}-J_{i, k}$. Then, either there is a negative circle connecting $i, j$ and $k$, or there is another configuration $\mathcal{O} 3$ such that $\mathcal{H}_{i}(\mathcal{O} 3)=\mathcal{H}_{i}(\mathcal{O} 1, \mathcal{O} 2)+J_{i, j}+J_{i, k}$. Which is a contradiction to the maximality of $\mathcal{O} 1$ and $\mathcal{O} 2$.

\subsection{Illustration of Stable and Unstable Systems}

Consider the example having no optimal configuration. 


\section{Example 1 (Unstable System)}

Consider again the traditional conflicting triangle of Spain, England and France as in Figure (1).

We feature the instability of the triangle from the perspective of the countries preference orders over the configurations.

Each configuration is of the form $S=\left(S_{E}, S_{S}, S_{F}\right)$. The 8 different configurations in total represent 4 different coalitions. The functions of the countries' gains which are $H_{E}=-3 S_{E} S_{S}-S_{E} S_{F}$, $H_{S}=-3 S_{S} S_{E}-2 S_{S} S_{F}$, and $H_{F}=-S_{F} S_{E}-2 S_{F} S_{S}$ produce the following equivalence classes of the countries:

The equivalence classes of $E$ :

$$
\begin{aligned}
& \Gamma_{E}=4: C_{E}^{4}=\{(-1,+1,+1),(+1,-1,-1)\}, \\
& \Gamma_{E}=2: C_{E}^{2}=\{(+1,-1,+1),(-1,+1,-1)\}, \\
& \Gamma_{E}=-2: C_{E}^{-2}=\{(+1,+1,-1),(-1,-1,+1)\}, \\
& \Gamma_{E}=-4: C_{E}^{-4}=\{(+1,+1,+1),(-1,-1,-1)\} .
\end{aligned}
$$

The equivalence classes of $S$ :

$$
\begin{aligned}
& \Gamma_{S}=5: C_{S}^{5}=\{(-1,+1,-1),(+1,-1,+1)\}, \\
& \Gamma_{S}=1: C_{S}^{1}=\{(+1,-1,-1),(-1,+1,+1)\}, \\
& \Gamma_{S}=-1: C_{S}^{-1}=\{(+1,+1,-1),(-1,-1,+1)\}, \\
& \Gamma_{S}=-5: C_{S}^{-5}=\{(+1,+1,+1),(-1,-1,-1)\} .
\end{aligned}
$$

The equivalence classes of $F$ :

$$
\begin{aligned}
& \Gamma_{F}=3: C_{F}^{3}=\{(-1,-1,+1),(+1,+1,-1)\}, \\
& \Gamma_{F}=1: C_{F}^{1}=\{(+1,-1,+1),(-1,+1,-1)\}, \\
& \Gamma_{F}=-1: C_{F}^{-1}=\{(-1,+1,+1),(+1,-1,-1)\}, \\
& \Gamma_{F}=-3: C_{F}^{-3}=\{(+1,+1,+1),(-1,-1,-1)\} .
\end{aligned}
$$

As we can see, there is no common maximal configuration in the ESF triangle since the intersection of the maximal equivalence classes of the three countries is $C_{E}^{4} \cap C_{S}^{5} \cap C_{F}^{3}=\emptyset$.

The system thus shifts between the individual maximal coalitions infinitely, driven by each country's endless search for its best position.

Figure (5) shows series of transitions in the triangle that cycles exhibiting the endless instability.

The progression starts from $S=(+1,+1,+1)$, which is the worst configuration to all the countries. At the first step, $E$ changes its state to -1 which yields the country an immediate benefit of $\Gamma_{E}=4$. This change shifts the countries to the equivalence classes $C_{E}^{4}, C_{S}^{1}$ and $C_{F}^{-1}$, respectively. At the second step, country $F$ make change and moves to $C_{F}^{1}$ equivalence class. Then, 

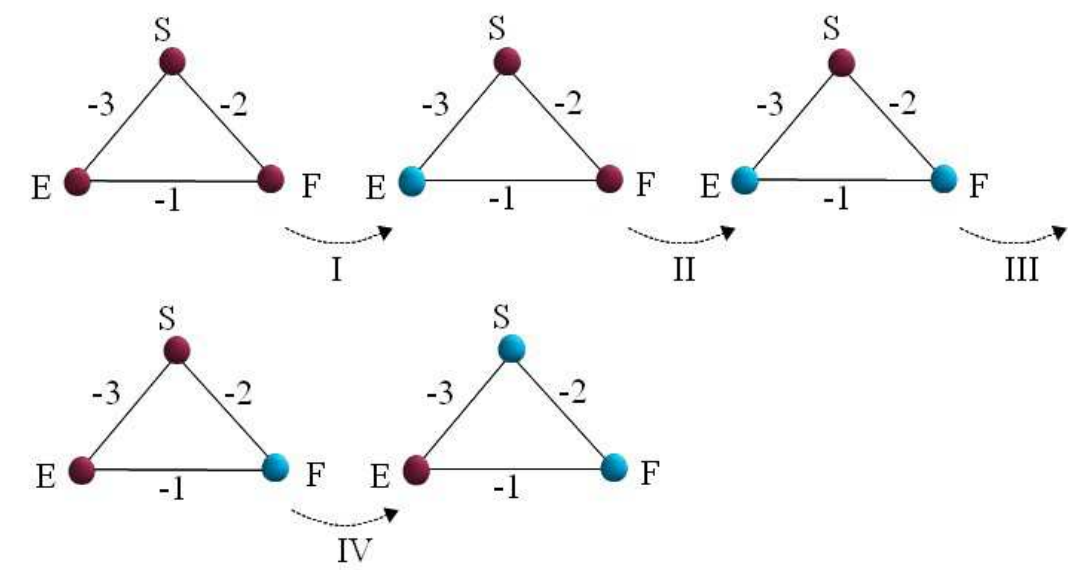

Figure 5: The diagram of successive configurations transition in the ESF conflicting triangle.

at the step III, E makes a change aimed to get back its best configuration in some further step. Finally, at the step $I V$, country $S$ changes to -1 , which brings the countries back to the original equivalence classes $C_{E}^{4}, C_{S}^{1}$ and $C_{F}^{-1}$.

Let us now turn to the example of a system where an optimal configuration exists.

\section{Example 2 (Stable System)}

A snap shot of the short period in the midst of war against Spain, when England and France were favorable to each others, illustrates a system where an optimal configuration exists (see Figure (6)).

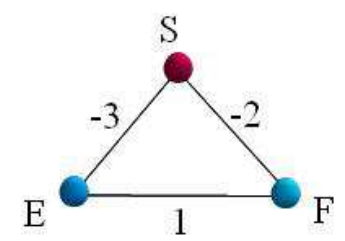

Figure 6: In this arrangement, the ESF triangle has an optimal configuration of coalitions $-\{S\}$, $\{E, F\}$, and thus is a stable system.

The maximal possible gains of $E, S$ and $F$ are $H_{E}=4, H_{S}=5$ and $H_{F}=3$, respectively, and the maximal equivalence classes of the countries coincide: $C_{E}^{4}=C_{S}^{5}=C_{F}^{3}=$ $\{(+1,-1,+1),(-1,+1,-1)\}$. Consequently, there are two optimal configurations in the system, $C_{E}^{4} \cap C_{S}^{5} \cap C_{3}^{2} \cap C_{F}^{3}=\{(+1,-1,+1),(-1,+1,-1)\}$, each of which guarantees its stability. 


\section{Rational Maximization of Countries}

Maximization of countries' gains is a rational process which can be defined in a general theoretical framework linking between the system's configurations information and the law of the configuration dynamics.

\subsection{Maximization As a Sequence of Individual Choices}

As we have mentioned, maximization is a sequence of the changes made by the countries that aim to improve their benefits. In our model, where the countries are individual actors possessing rationality, a change is made by the rational components such as the observation and the decision making.

Let us examine the mechanisms that stand behind the changes. At any configuration $S$, a country $i$ can make a choice for its state. The alternative is either to keep the current state, or to invert it and thus to move to configuration $S^{-i}$. Making the choice consists of two successive phases - observations and decision making. The phase of observation involves featuring the equivalence classes and looking for transitions between the configurations aiming at the immediate or the further improvements of the gain. The phase of the decision making, in turn, involves the selection of an immediate reply based on the results of the observation phase. Denote by $O_{i}(S)$ the set of desirable configurations and by $D_{i}(S)$ the immediate reply, $D_{i}(S) \in\left\{S, S^{-i}\right\}$.

The decision making phase can be based either on the best reply principle, which accepts the choices that improve the gain immediately, or on the forecast principle, which takes into account the choices that improve the gain in a further step. The choice between the equivalent alternatives can be based either on the principle of random move or on the principle of anticipation of feedback from the others countries.

The following example illustrates observation and decision making by the countries of the ESF conflicting triangle in the series of transition of configurations.

\section{Example 3}

Figure (7) shows several possible configuration transitions in the conflicting triangle as the result of particular combinations of observations and decisions made by the countries in the ESF triangle.

The initial configuration $S=(+1,+1,-1)$ belongs to the equivalence classes $C_{E}^{-2}, C_{S}^{-1}$ and $C_{F}^{3}$ of $E, S$ and $F$, respectively. Let us analyze the $I-I V$ transitions shown in the Figure (7).

$T_{I}$ : Observation by $E$ results in $O_{E}(S)=\{(+1,+1,-1),(-1,+1,-1)\}$ where configuration $(-1,+1,-1)$ is for the immediate improvement and $(+1,+1,-1)$ is for the improvement in a further step, which will eventually lead to the $E$ 's maximal configuration $(+1,-1,-1)$. $E$ makes the decision $D_{E}(S)=(-1,+1,-1)$ and thus moves the system to the equivalence classes $C_{E}^{2}, C_{S}^{5}$ and $C_{F}^{1}$. 


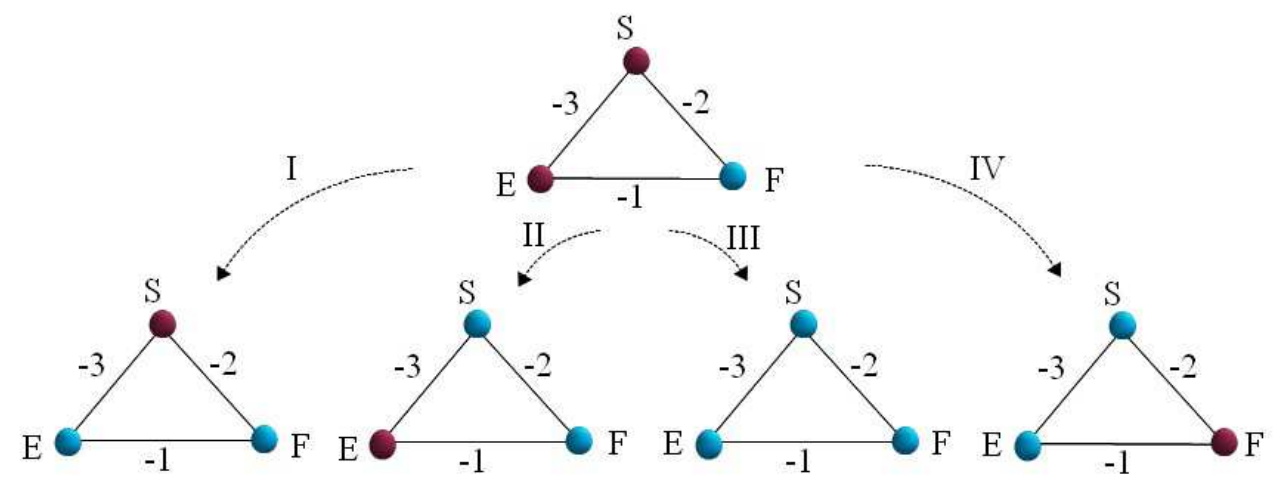

Figure 7: The diagram of transitions of configurations prompted by the decisions in the ESF conflicting triangle.

Note that, while the change satisfies only country $S$, it is positive for the system as a whole; the new configuration is the system's maximum.

$T_{I I}$ : If the change is done by country $S$ who makes the decision $D_{S}(S)=(+1,-1,-1)$, the new configuration belongs to the classes $C_{E}^{4}, C_{S}^{1}$ and $C_{F}^{-1}$.

Though the configuration improves the gain of the system itself, it is not the system's maximum.

$T_{I I I}$ : If the change is performed by both the countries $E$ and $S$ simultaneously, with $D_{E}(S)=$ $(-1,+1,-1)$ and $D_{S}(S)=(+1,-1,-1)$, the resulting configuration is $S=(-1,-1,-1)$ which belongs to the equivalence classes $C_{E}^{-4}, C_{S}^{-5}, C_{F}^{-1}$.

This is the worst case for each of the countries, as well as for the system itself.

$T_{I V}$ : When the changes are performed by all the countries $E, S$ and $F$ simultaneously, and the resulting configuration $(-1,-1,+1)$ belongs to the initial equivalence classes $C_{E}^{-2}, C_{S}^{-1}$ and $C_{F}^{3}$

It is worth to emphasize that since each country at any time can decide to update or not to update its state, the transitions are all equiprobable. All the transitions are codified in the configurations information trees described below.

\subsection{Configurations Information Tree}

From the point of view of a given country, the choices made by the others are the direct or indirect outcomes of the country's own decision. Such dependencies between the country's choice and the decision of the others, while developing staring from the initial configuration, define a tree-like structure. 
We define the configurations information tree as the centralized structure containing all the possible system's configurations and all the possible transitions between them. The tree of a country $i$ is built as follows. At the root there is the initial configuration $S$ of the system. The root splits into two choice nodes corresponding to the two possible choices of the country $+S_{i}$ and $-S_{i}$. Each of the nodes splits into $2^{N-1}$ configuration nodes, which are the possible decisions by the other countries. Starting from the configuration nodes, the tree develops the same way as it does from the root. The choice nodes of the tree continue to develop until they correspond to a configuration that has already appeared in a configuration node of the tree.

The information tree contains the transitions of any feasible maximization processes prompted by the country. It allows the country to make unlimited observations and to build the best reply strategy starting from any configuration. Let us look at the information tree of England in the ESF conflicting triangle of Figure (1).

\section{Example 4 (Information Tree of England in the ESF Conflicting Triangle)}

Figure (8) shows the information tree of $E$. The configuration nodes are marked by red (dark) color until they or their inverses reappear in the tree, then they are marked by green (light) color. The values of corresponding countries' gains are shown next to each configuration node. The choice nodes are marked by light blue.

The lines proceeding from a configuration node correspond to the choices made by $E$ and the dashed lines drawn from the choice nodes represent the transitions initiated by the others.

Observation function includes a search for the pathes in the tree that brings to a best configuration. The size of the tree defines the lower bound on the complexity of the search, which is a lower bounds on the minimum amount of time required by the most efficient search algorithm. Since only one among any two symmetric configurations is developed, and each of the respective configuration nodes is followed by the two choice nodes, the tree contains $\mathcal{O}\left(2^{N}\right)$ nodes. It follows that the rational functions get harder exponentially as the system grows.

\section{A Formal Implementation of Rational Maximization}

A system of countries represents a decentralized multi-agent system of individual actors separated by geographical and cultural distances. The geographical location and the initial conditions foreordain different capacities and different degrees of impact by technological development. Those distances and differences result in diverse levels of exposure to the system information, as well as in divers technical capacities of observation. The differences become more distinct with the increase of the system's size.

The complete system information available to a country and its extensive technical capacities contrast with the limited system knowledge and limited capacity. The limited knowledge of a country prevents from proper observation of the other's states and decisions. The limited capacity 


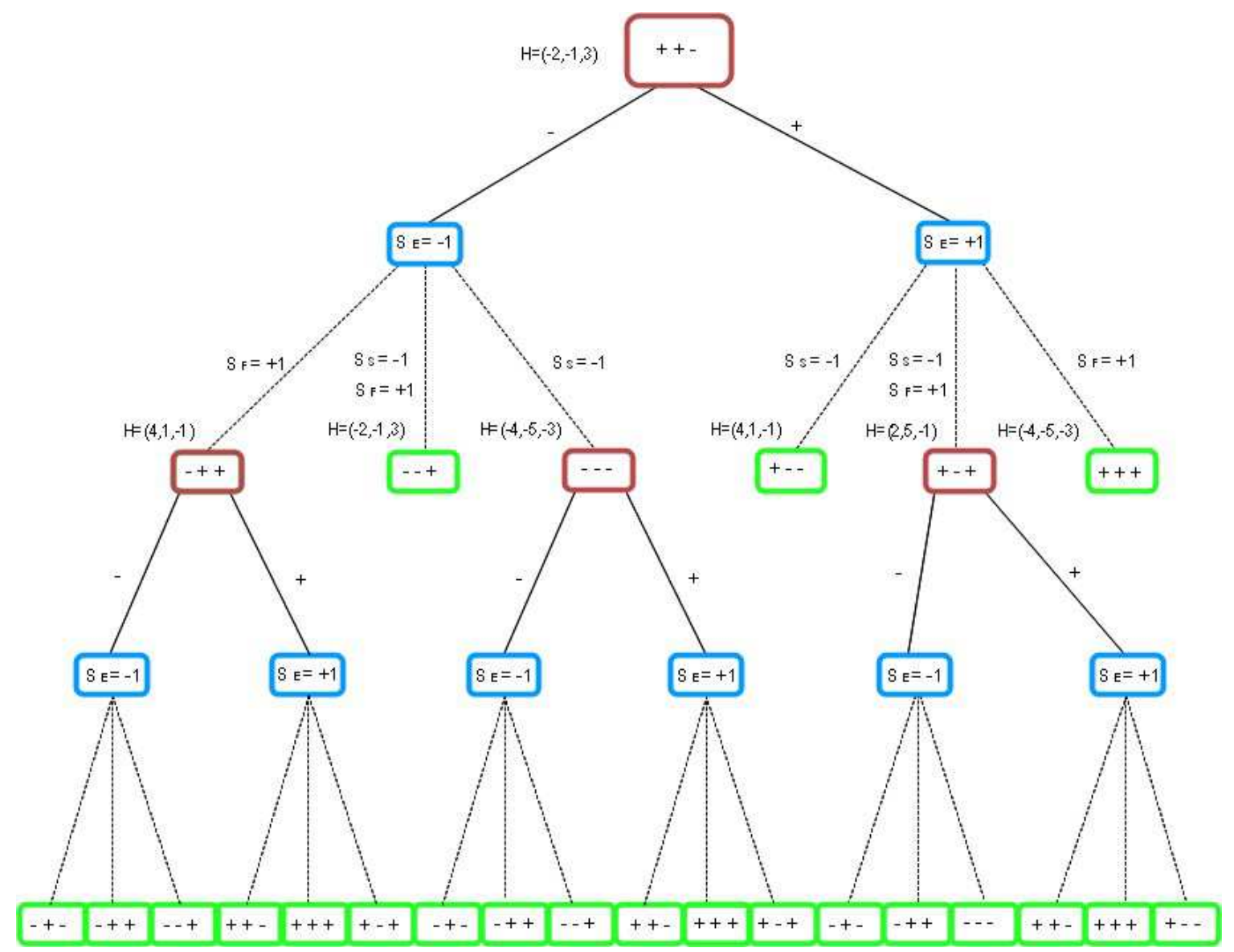

Figure 8: Configurations Information tree of England in the ESF conflicting triangle. In the tree, the configuration nodes are alternated with the choice nodes followed by the decision of the country. The root represents the initial configuration.

restricts its ability to build the system information and to track the maximization processes. Both limitations perturb the observation function of the country introducing limitations in their rationality.

Another restriction in this concern is the latency in the decision making which affects the quality of the decision output. Here, we ignore the decision latency as an issue of a different subject.

The following cases can be distinguished in the ranges of the classification of capacities.

I: Imagine a system which has an optimal coalitions and where the countries have extensive technical capacities. The maximization process in the system is in fact the optimization. A correct information, the accurate configurations data guarantee the appropriate observation and decision making and thus are crucial for the optimization.

The existence of unique optimum combined with the countries' extensive technical capacities guarantees a rapid stabilization with hight probability. However, systems that stabilize spon- 
taneously are rare. The probability that the system has an optimum vanish exponentially with the size.

$I I$ : The system with no optimum is more likely. This case, combined with the rational instability, produces the infinite competition for beneficial coalitions that trap the maximization process into an infinite cycle.

$I I I$ : Finally, the most real situation is the system with no optimum where some of the countries have limited system knowledge and a limited capacity. The rationality limitations influence the maximization which either involves an infinite competition or becomes a finite stabilization. The latter lead to a stable state which though maybe temporary. This case is investigated in the next section.

\subsection{Rationality Limitations and Information Manipulation}

In order to attain the maximal benefit with high probability a country have to be able to forecast the behavior of the others. The forecast principle consists of screening the possible configuration transitions that can eventually lead to the country's maximal gain.

It is not enough for a country to be only aware of its neighbors. In order to complete a proper forecast, the country needs to keep in view all the dependencies and bonds of the other countries linked to it indirectly. In practice, the forecast screening bases on the logic of the configurations information tree. Therefore, completeness of the information tree of the country defines the completeness of its forecasts ability.

As a result of the limited system knowledge and the limited capacity, the country construct an incomplete. However, it is not only the limited rationality that confines the forecast abilities. The configuration information trees grow exponentially following the system's size growth. As a result, a complete tree gets harder to build, so harder it gets to forecast.

The countries with a limited system knowledge and a limited capacity are unable to build the complete information trees. Therefore, they are unable to perform a proper forecast of the possible transitions. As a result, the limited system knowledge and the limited capacity produce such rationality limitations that, even with a good will, the countries tend to make wrong choices. The countries may neither know if there is a common maximum, nor be able to forecast the common behavior and make the corresponding choices.

In practice, in cases of the limited rationality, the rationality itself ceases to be the criterium of choices and is substituted by inspirations of all kind, such as religious, moral or cultural codes.

Therefore, two coexisting types of countries can be distinguished in such systems: 1) countries possessing the complete system information and extensive capacities, 2) countries who has a limited system knowledge and a limited capacity.

While the knowledgeable countries forecast and make choices that should benefit them in a 
future step, the limited countries either make the changes bearing immediate profit or follow the inspirations and the cultural codes. Not having knowledge necessary to conclude on existence of a common maximum and not being able to forecast the future steps, the secondary actors are interested to stay within the set of their local maximums. The countries adhere to the policy where they undertake changes only upon an immediate improvement.

It is reasonable to assume that inspirations and cultural codes playing a centralizing role on the limited countries can be imposed by the knowledgeable ones that aims to achieve their benefits. Such an influence is the essence of information manipulation phenomena.

On this basis, we refer the countries possessing the complete system information and extensive capacities as primary actors, and the countries who has a limited system knowledge and a limited capacity as secondary actors.

The information manipulation can be described as acting to provide wrong or partial information while pursuing certain objectives. The information manipulation can be viewed as a natural consequence of the extended rationality of the primary actors.

The combination of knowledge and ignorance may bring to stabilization of the systems despite of the negative circles. Below we illustrate the system that stabilizes regardless of lack of the optimum.

\section{Example 5 (Information Manipulation and Non-optimal Stability)}

Here, we present a system of four countries (see figure (9)) forming two triangles, a stable and an unstable, where the latest is identical to the ESF conflicting triangle.

Assume that country 1 is a primary actor who possesses the complete system information and the extensive capacities, while all the others are the secondary actors having limited system knowledge and limited capacities. The primary actor's node is emphasized by the additional circle around the nodes.

The initial configuration is only profitable to country 3 . Therefore, country 1 initiates a maximization process by first inverting its state to +1 (step I), and then convincing 2 to invert it's state too asserting that 3 going to switch to $S_{3}=-1$ (step II). As a result, country 3 changes to $S_{3}=-1$ seeking an immediate improvement. The resulting configuration is the maximal one for 1 and is the stable one long country 3 is unable to lead a profitable maximization process.

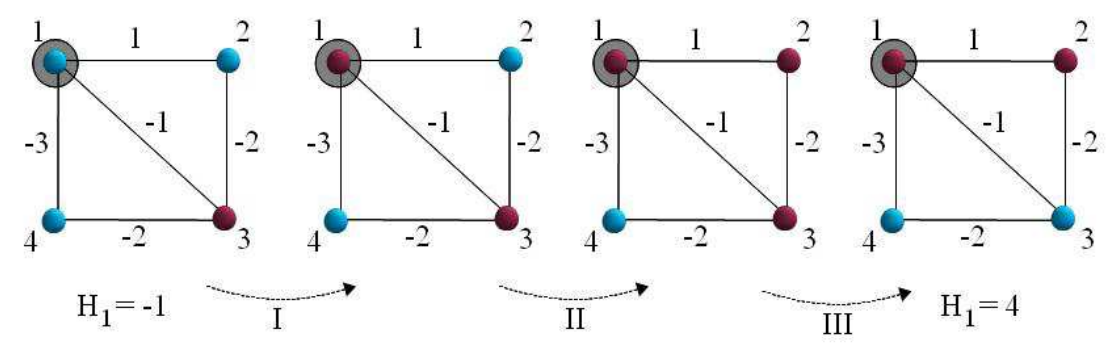

Figure 9: Stabilization of a system having a negative circle by the information manipulation. 
The unlimited rationality give to the primary actor (or to group of leading countries with their own common maximum) an advantage over the others to govern changes leading to profitable coalitions. The widespread belief that keeping the information is secret benefits country is fully justified in this case.

However, as soon as a secondary actor supplements the lack of the information with the data gained by tracking the maximization history or from the information exchange, the non-optimal stability breaks. Therefore, a non-optimal stable state is rather a temporary one, as it is seen in history.

The picture changes when an optimum exists. Once the existence is known, the complete information is the common interest of the countries. In this case, the contrary to the widespread belief about advantage of keeping information secret is valid: disclosing information helps the countries to achieve the common profitable coalitions.

The phenomena of the information manipulation is intrinsic to any system of individual actors where the respective capacities vary. As we have noted, the frame of the natural model is applicable to the large spectrum of social, political or economics domains, from the dynamics of social opinion to the dynamics of interactions between publics and private banks.

\subsection{Simulation of Coalition Forming in a Finite-size System}

To illustrate the coalition forming, we created a graphical computer simulation of the natural model. The simulation is based on the structure of the model, where the choice making process consists of phases of observation and decision, as defined earlier. The countries are represented by the simultaneously running threads whose action readiness grows with the countries rationality. There is no predefined order on the countries' decision making.

For the sake of simplification in simulation of individual actors, country's observation function, instead of searching for the best paths, is reduced to determination of state bring an immediate gain. Then, with regards to the type of the actor, the state or its inverse is adopted by the actor. The primary actors make a disadvantageous change aimed put the system out of its local maximum and to improve the gains in further steps. Such strategy enables us to fully simulate a maximization process by following randomly the equiprobable chains of configuration transitions.

In the simulation, the primary actors randomly make the choices due to an expected gain. The secondary actors undertake only changes bearing an immediate gain. They adhere to the anticipation of a negative result that provides a benefit without jeopardizing the whole system. This implies that no rational instability is triggered by the secondary actors, as well as the no-cost changes.

Here, we present the simulation of coalition forming in a finite-size system that involves both the primary and the secondary actors. The system in its initial state is shown in figure (10). 


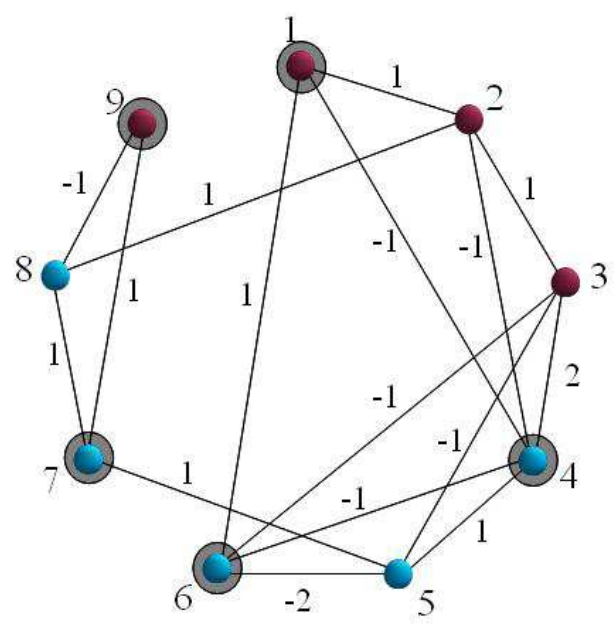

Figure 10: The initial state of a system of 9 countries. Countries countries 1, 4, 6, 7 and 9 are the primary actors, and 2, 3,5 and 8 are the secondary actors. Two groups with disconnected primary actors are formed in the system.

Primary actors are countries 1, 4,6 and 7,9. The countries are emphasized by the additional circles around the nodes in the figure. The remaining countries are the secondary actors.

The primary actors countries form two groups $\{1,4,6\}$ and $\{7,9\}$ which have no direct connection between them.

Both groups have the local common maximums:

$$
\mathcal{S}_{1,4,6}=\{(1,-1,1),(-1,1,-1)\} \text { and } \mathcal{S}_{7,9}=\{(1,1),(-1,-1)\}
$$

The negative circles of the system are $\{2,3,4\},\{1,2,3,4\},\{3,4,5\},\{3,4,6\},\{1,2,3,4,5,6\}$ and $\{7,8,9\}$. As it can be observed, the system consists of two parts, each of which formes a negative circle, the right part $\{1,2,3,4,5,6\}$ and the left part $\{7,8,9\}$.

Despite the fact that the right part contains several negative circles, it is being stabilized due to particular interactions between primary and secondary actors (see the stabilization in Figure (11)). The right part of the system is stable in the last configuration 15 of the figure: the group of primary actors 1, 4,6 meet their common maximum, and none of the secondary actors' changes can give an immediate improvement. 
1.

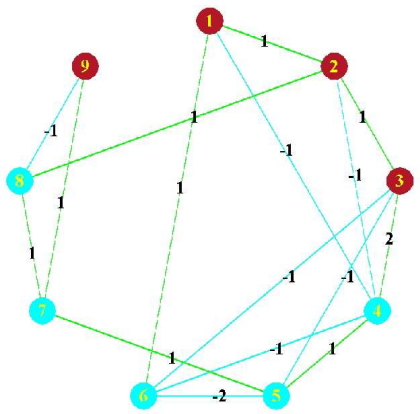

4.

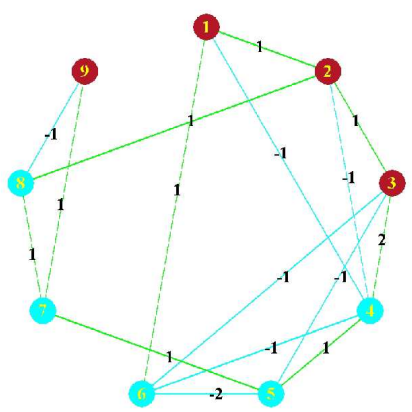

7.

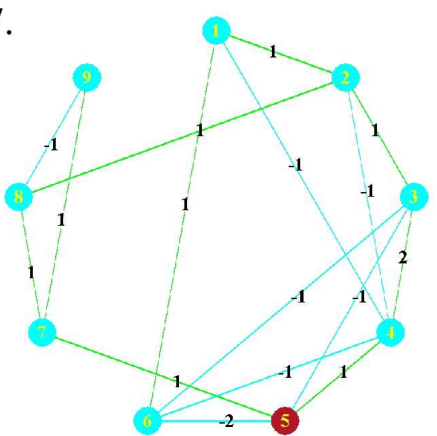

10.

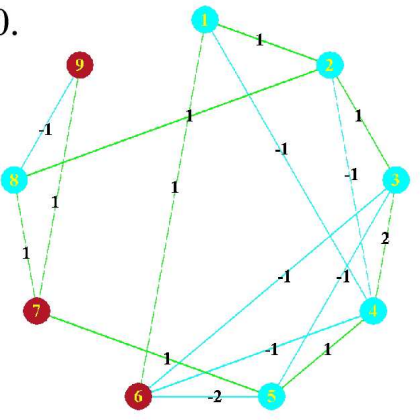

13.

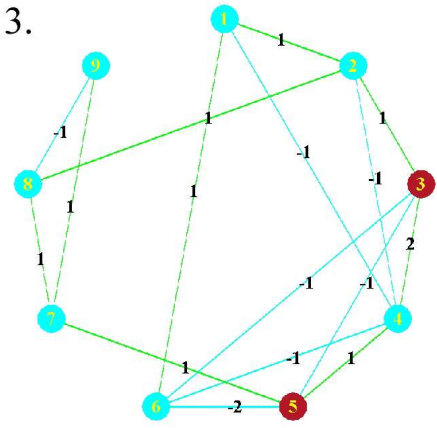

2.

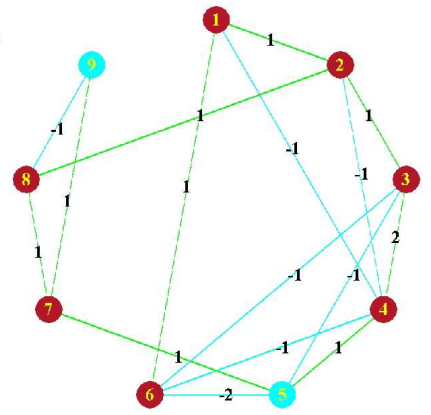

5.

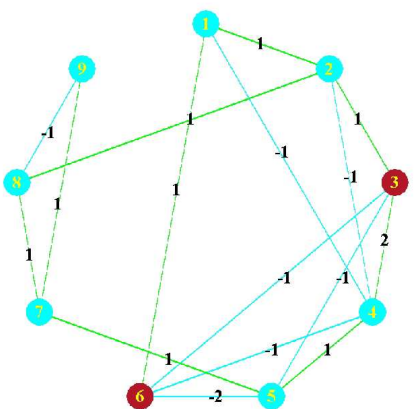

8.

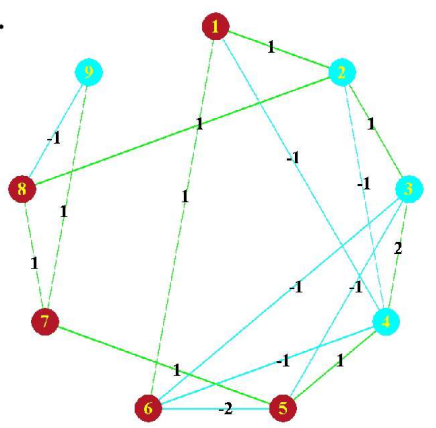

11.

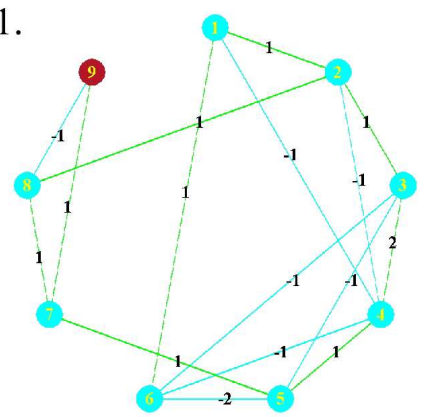

14.

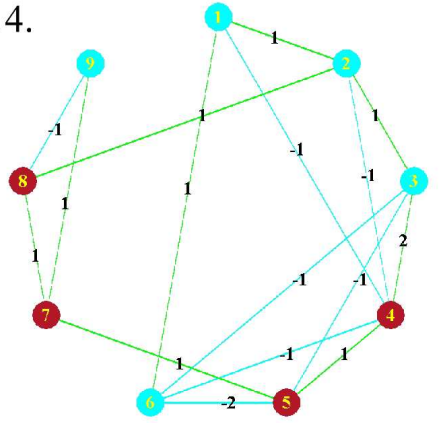

3.

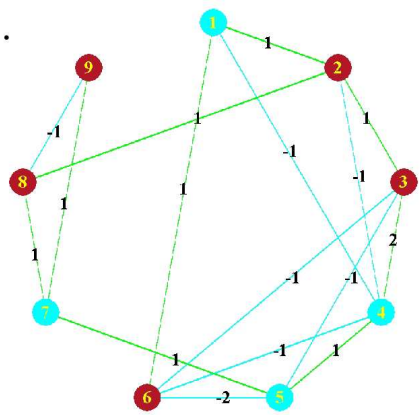

6.

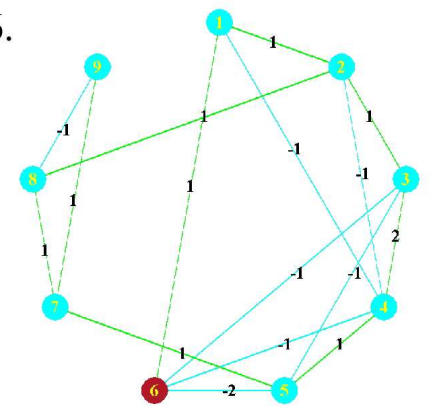

9.

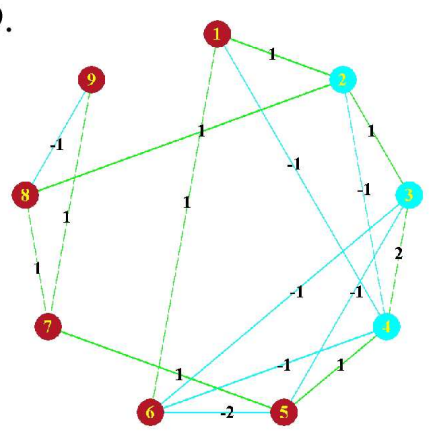

12.

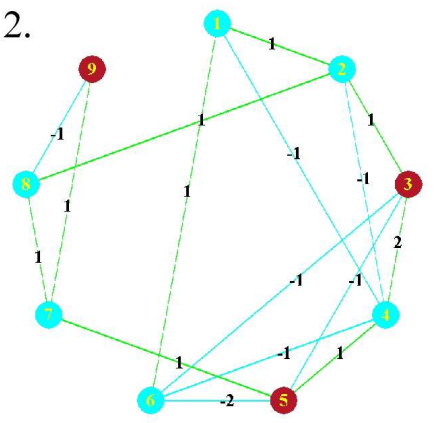

15.

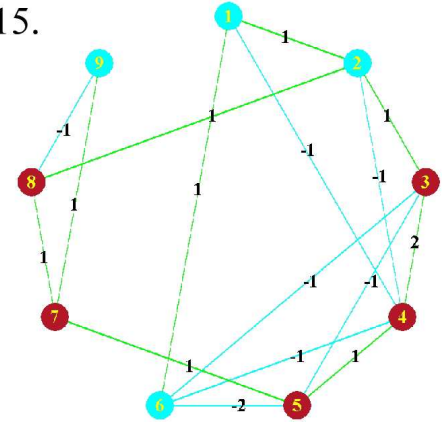

Figure 11: Simulation of the coalition forming -15 steps of stabilization of the right part. 
Let us look at the stabilization in details:

1. The initial state.

2. The principal actors 4 and 6 simultaneously make an immediate improvement of the gain time, the principal actors 7 and 9 make the change for an expected gain, and 8 improves its gain in reply.

3. The principal actor 1 changes for a future gain and 4 improves its gain, 7 and 9 repeats the previous change.

4. Country 1 improves its gain, 6 makes a change for a future gain and 8 improves its gain.

5. Country 6 improves its gain, 1 makes a simultaneous change for an expected gain, 2 improves its gain in reply.

6. Country 3 improves its gain in result.

7. Country 6 makes a change for an expected gain and 5 improves its gain in reply.

8. Country 1 makes a change for an expected gain and 6 improves as result, 7 makes the change for a future gain and 8 improves in result.

9. Country 9 makes the change for an expected gain.

10. Countries 1, 5 and 8 improve their gains.

11. Countries 6 and 7 make the change for their expected gains.

12. Countries 5 improves its gain.

13. Country 9 makes the change for a future gain.

14. Countries 3 and 4 improve their gains, 7 makes the change for a future gain and 8 improves as result.

15. Countries 3 improves its gain.

In the given disposition, the stability of the right part is solid enough not to be broken despite of the fluctuation in the left part. Figure (12) ) shows the instability of the triangle $\{7,8,9\}$, from the step 16 to the step 22 . 
16.

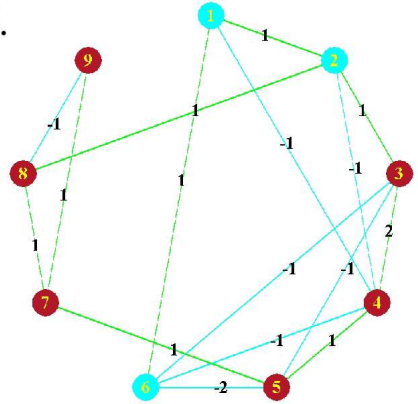

19.

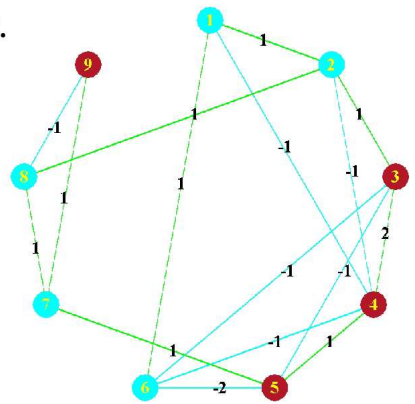

17

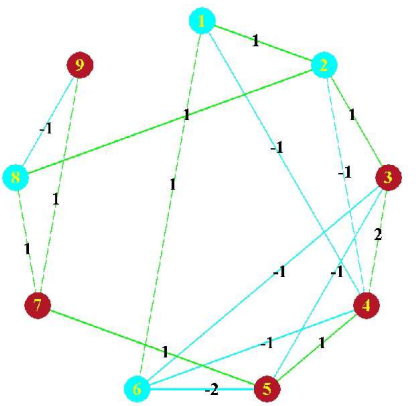

20.

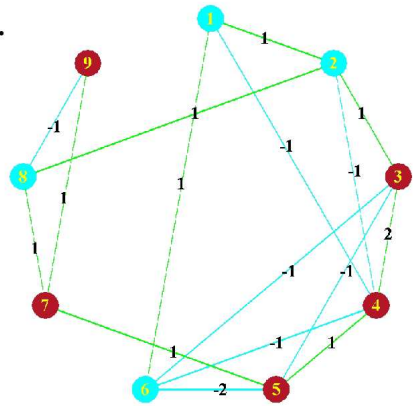

18.

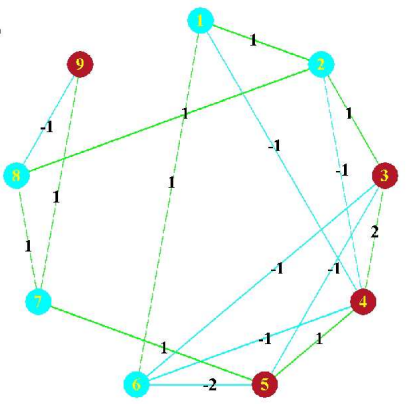

21.

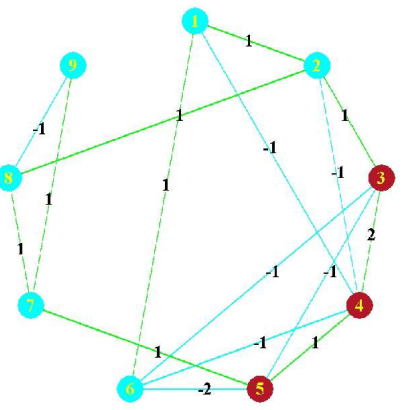

Figure 12: Partial instability - the instability of the left part versus the stability of the right part.

However, the stability of $\{1,2,3,4,5,6\}$ is fragile since it breaks even upon a slight change of a propensity between the secondary actors. For instance, if the propensity between the countries 2 and 8 rises to 2 , the instability of the left part is propagated to the stable right part. Another example is when the propensity between countries 3 and 5 decreases to -3 . Then, country 3 will be always able to improve its gain immediately from -1 to 1 by inverting its choice.

\section{Conclusions and Remarks}

The historical examples such as the conflicting triangle of England, Spain and France, the countries of the whole European Union, the Soviet and the Western camps illustrate the existence of instability in the formation of coalitions during a significant period of their history.

The mapping from history to the natural model allows us to reproduce and provide an explanation of these instabilities and the cycling of coalitions. It then enable us to analyze the conditions necessary to the system's stability.

Overall, achieving optimal or stable coalitions requires a significant computation power and a complete availability of the information. All types of rationality limitations impact the stabilization and optimization processes.

It can be seen from our study of the model that, as the system's complexity grows along with the connexity and the size, the stable or the optimal state become harder to achieve. To overcome those difficulties, the large systems (such as the United States of America) tend to subdivide 
themselves into small weakly connected sub-systems that manage their internal optimizations and stabilizations. Large systems that do not follow this course (the Soviet Union, for example) face difficulties that come from the complex instabilities communicated to all its parts via the strong interrelations in the system.

However, a spontaneous maximization that exhibits the bottom-up dynamics of coalition forming is rare to stabilize. The probability that the system becomes stable vanishes exponentially with the size. As a future research, we aim to consider an amalgam of both top-down and bottom-up dynamics of the coalition forming. The stabilization by means of an external field could produce such an apposite amalgam. The external field, while polarizing the interests of the countries, leads to the emergence of new opposing alliances. The countries, attaching themselves to one or to the other, find new interests that unite or separate them based on a pragmatic motivation instead of the historical concerns. Hereby, the external field model enables the stabilization among the countries while keeping the short range character of the interactions between them. This insight will be investigated in a forthcoming paper.

\section{References}

Binder, K. \& Young, A.P. (1986). Spin-Glasses: experimental facts, theoretical concepts, and open questions, Review of Modern Physics, 58, 801-911

Toulouse, G. (1977). Theory of the frustration effect in Spin Glasses: I, Comm. on Physics, 2

Galam, S. \& Moscovici, S. (1991). Towards A Theory Of Collective Phenomena: Concensus And Attitude Changes In Groups, European Journal Of Social Psychology, 21, 49-74

Axelrod, R. \& Bennett, D.S. (1993). A landscape theory of aggregation, British Journal Political Sciences, 23, 211-233

Florian, R. \& Galam, S. (1993). Optimizing conflicts in the formation of strategic alliances, Eur. Phys. J. B , 16, 189-194

Galam, S. (1998). Comment on A landscape theory of aggregation, British Journal Political Sciences, $28,411-412$

Galam S. (2002). Spontaneous Coalition Forming. Why Some Are Stable?, Springer-Verlag Berlin Heidelberg 2002, Springer-Verlag Berlin Heidelberg 2002, S. Bandini, B. Chopard, and M. Tomassini (Eds.):ACRI 2002, LNCS 2493, 1-9

Galam S. (1996). Fragmentation Versus Stability In Bimodal Coalitions, Physica, A, 230, 174-188

Gerardo G. N. \& Samaniego-Steta F. \& del Castillo-Mussot M. \& Vazquez (2007). Three-body interactions in sociophysics and their role in coalition forming, Physica, A, 379, 226-234 
Vinogradova G. (2012). Correction of Dynamical Network's Viability by Decentralization by Price, Journal of Complex Systems, 20, 1, 37-55

Van Hemmen J.L. (1982). Classical Spin-Glass Model, Physical Review Letters, 49, 6,

Tim Hatamian G. (2005). On alliance prediction by energy minimization, neutrality and separation of players, arxiv.org/pdf/physics/0507017

Matthews R. (2000). A Spin Glass model of decisions in organizations, Business Research Yearbook, G. Biberman, A. Alkhafaji (eds), Saline, Michigan: McNaughton and Gunn, 7, 6

Galam S. \& Gefen Y. \& Shapir Y. (1982). Sociophysics: A new approach of sociological collective behavior, British Journal Political Sciences, 9, 1-13.

Antal T. \& Krapivsky P.L. \& Redner S. (2006). Sociophysics: A new approach of sociological collective behavior, Physica D, 224, 130136.

Acemoglu D. \& Egorov G. \& Sonin K. (2006). Dynamics and Stability of Constitutions, Coalitions, and Clubs, American Economic Review, American Economic Association, 102(4), 46-76. 


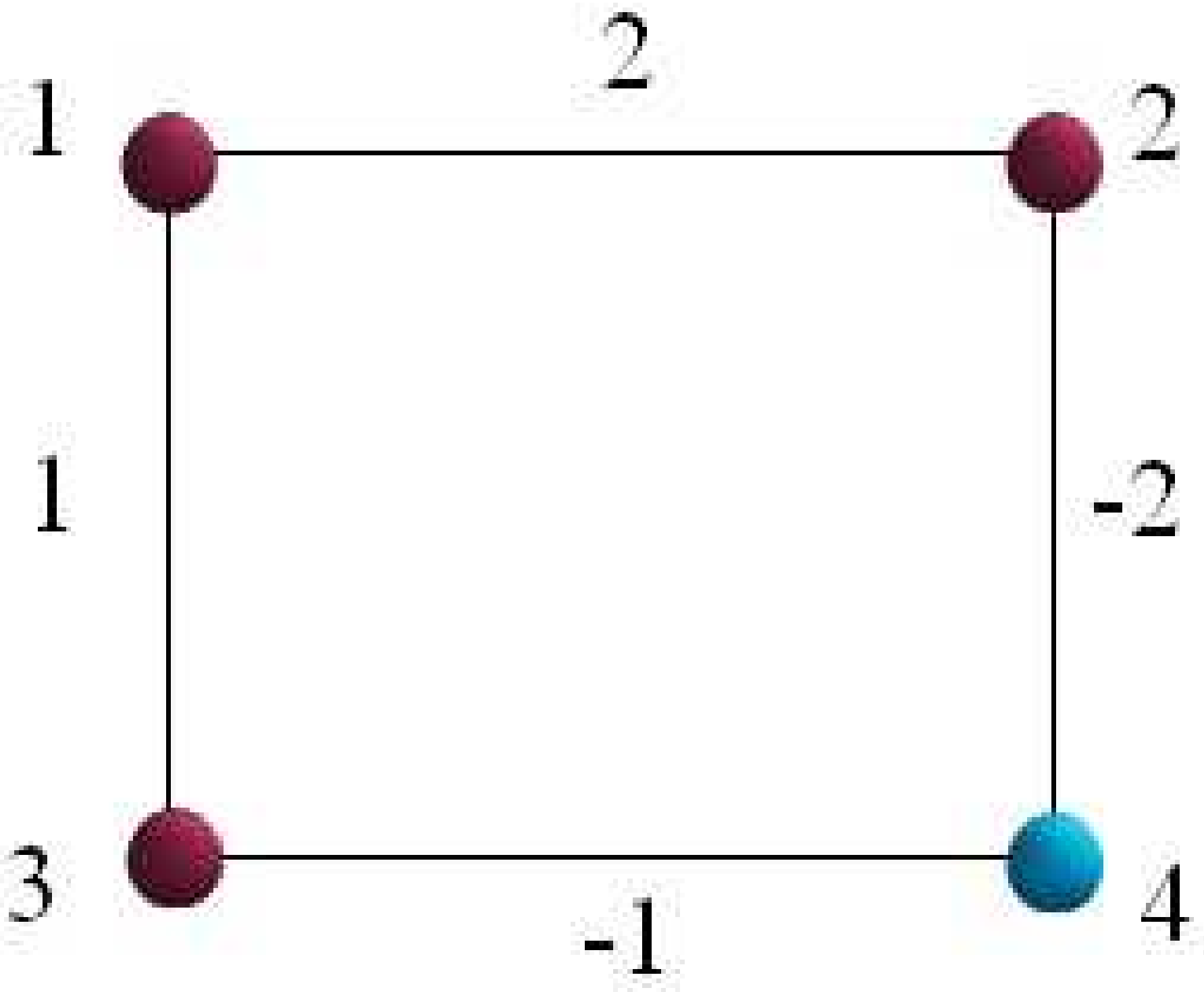




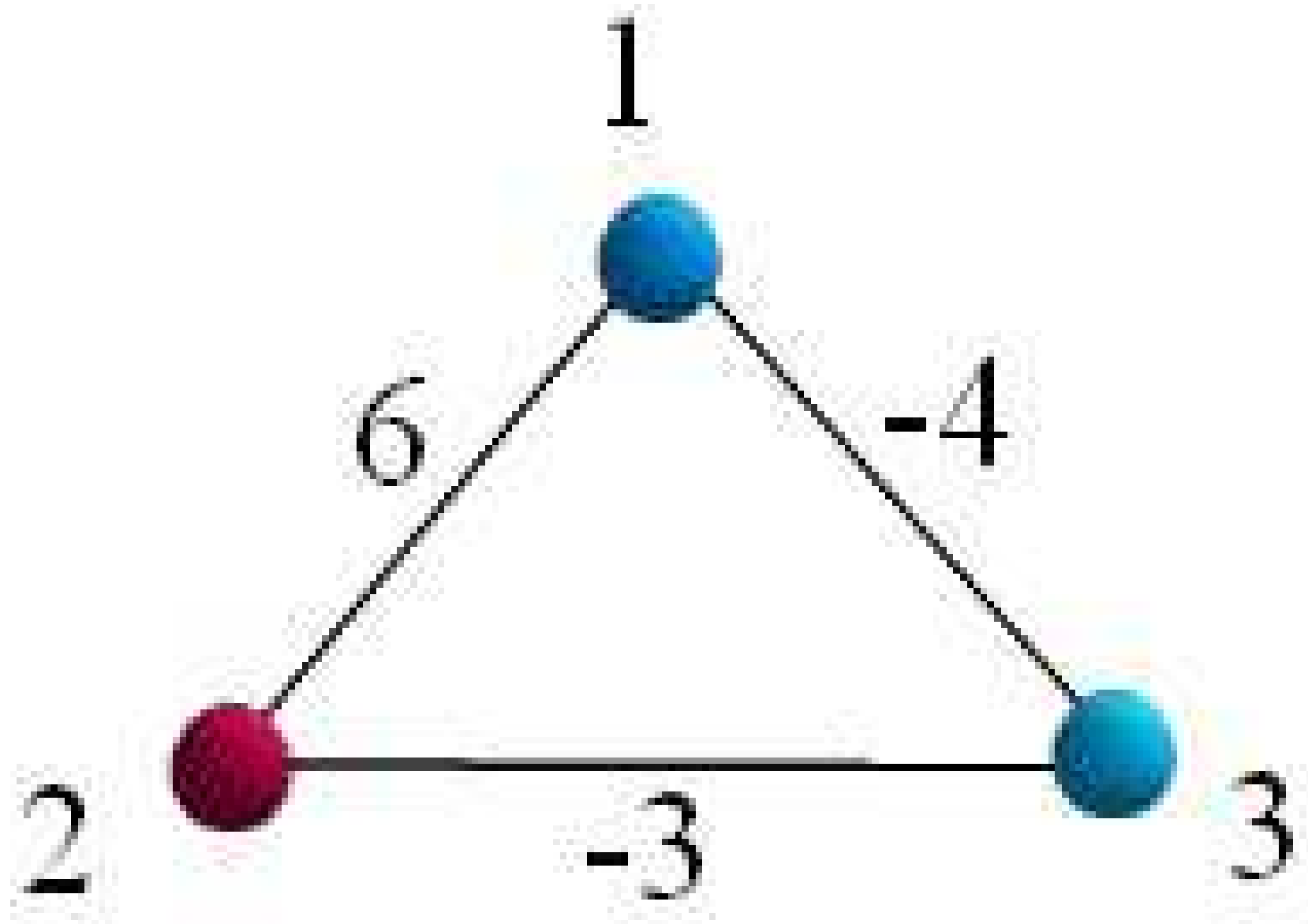

